\section{Temporal dynamics of the pharmacological MRI response to subanaesthetic ketamine in healthy volunteers: A simultaneous EEG/fMRI study}

\author{
Rebecca McMillan ${ }^{1}\left(\mathbb{D}\right.$, Anna Forsyth ${ }^{1}$, Doug Campbell ${ }^{2}$, \\ Gemma Malpas'2, Elizabeth Maxwell ${ }^{2}$, Juergen Dukartt ${ }^{3,4,5}$, \\ Joerg F Hipp ${ }^{3}$ and Suresh Muthukumaraswamy ${ }^{1}$
}

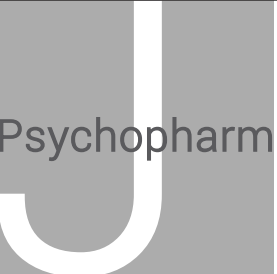

Journal of Psychopharmacology $1-11$

(c) The Author(s) 2019 Article reuse guidelines: sagepub.com/journals-permissions DOI: $10.1177 / 0269881118822263$ journals.sagepub.com/home/jop

(A) SAGE

\begin{abstract}
Background: Pharmacological magnetic resonance imaging has been used to investigate the neural effects of subanaesthetic ketamine in healthy volunteers. However, the effect of ketamine has been modelled with a single time course and without consideration of physiological noise.

Aims: This study aimed to investigate ketamine-induced alterations in resting neural activity using conventional pharmacological magnetic resonance imaging analysis techniques with physiological noise correction, and a novel analysis utilising simultaneously recorded electroencephalography data. Methods: Simultaneous electroencephalography/functional magnetic resonance imaging and physiological data were collected from 30 healthy male participants before and during a subanaesthetic intravenous ketamine infusion.

Results: Consistent with previous literature, we show widespread cortical blood-oxygen-level dependent signal increases and decreased blood-oxygenlevel dependent signals in the subgenual anterior cingulate cortex following ketamine. However, the latter effect was attenuated by the inclusion of motion regressors and physiological correction in the model. In a novel analysis, we modelled the pharmacological magnetic resonance imaging response with the power time series of seven electroencephalography frequency bands. This showed evidence for distinct temporal time courses of neural responses to ketamine. No electroencephalography power time series correlated with decreased blood-oxygen-level dependent signal in the subgenual anterior cingulate cortex.

Conclusions: We suggest the decrease in blood-oxygen-level dependent signals in the subgenual anterior cingulate cortex typically seen in the literature is the result of physiological noise, in particular cardiac pulsatility. Furthermore, modelling the pharmacological magnetic resonance imaging response with a single temporal model does not completely capture the full spectrum of neuronal dynamics. The use of electroencephalography regressors to model the response can increase confidence that the pharmacological magnetic resonance imaging is directly related to underlying neural activity.
\end{abstract}

Keywords

Ketamine, pharmacological MRI, simultaneous EEG/FMRI

\section{Introduction}

Ketamine is a non-competitive N-methyl-D-aspartate receptor antagonist that has traditionally been used in psychiatric research for its ability to mimic the symptoms of schizophrenia (see Frohlich and Van Horn (2014) for review) and is used clinically as an anaesthetic. However, more recently, ketamine has received considerable attention due to its rapid antidepressant effect at subanaesthetic doses (see Abdallah et al. (2015) for review). Consequently, development of an understanding of the neural effects of ketamine is of growing interest in both the understanding of the neurobiology of major depressive disorder (MDD) and in the development of novel antidepressants. There is a growing body of literature examining the effects of subanaesthetic doses of ketamine on the brain using pharmacological magnetic resonance imaging (phMRI) in healthy volunteers (Deakin et al., 2008; De Simoni et al., 2013; Doyle et al., 2013; Höflich et al., 2016; Javitt et al., 2018; Stone et al., 2015).

phMRI is a functional imaging analysis technique that uses the blood-oxygenation-level dependent (BOLD) signal measured with functional MRI (fMRI) to index the modulatory effects of psychopharmacological compounds during a resting-state scan. This mass univariate approach has been applied to ketamine with a focus on slow neural responses that follow a time course similar

\footnotetext{
${ }^{1}$ School of Pharmacy, Faculty of Medical and Health Sciences, University of Auckland, Auckland, New Zealand

2Department of Anaesthesiology, Auckland District Health Board, Auckland, New Zealand

${ }^{3}$ F. Hoffmann-La Roche, Pharma Research and Early Development, Roche Innovation Centre Basel, Basel, Switzerland

4Institute of Neuroscience and Medicine, Brain \& Behaviour (INM-7), Research Centre Jülich, Jülich, Germany

${ }^{5}$ Institute of Systems Neuroscience, Medical Faculty, Heinrich Heine University Düsseldorf, Düsseldorf, Germany
}

Corresponding author:

Rebecca McMillan, School of Pharmacy, Faculty of Medical and Health Sciences, Private Bag 92019, Auckland 1142, New Zealand.

Email:r.mcmillan@auckland.ac.nz 
to that of ketamine's pharmacokinetics. In their seminal work, Deakin et al. (2008) used a pseudoblock analysis approach to investigate the effects of ketamine on the BOLD signal as an alternative to techniques using a single psychological, physiological or pharmacokinetic regressor to detect the effects of ketamine, as these techniques risk missing brain activity occurring on a different time course. The pseudoblock analysis showed ketamine-induced increases in BOLD signal in the mid-posterior cingulate cortex (PCC), thalamus and temporal cortical regions, with decreased in BOLD signal in the orbitofrontal cortex and the subgenual anterior cingulate cortex (sgACC). Furthermore, the time courses of these changes differed across regions. Most activity peaked between 3 and 5 minutes following the start of the ketamine infusion, before returning towards baseline. A notable exception was the decrease in BOLD signal in the subgenual cingulate cortex, which remained significantly decreased at 7 minutes post-infusion. As the sgACC is overactive in MDD and effective antidepressant treatment is associated with a decrease in sgACC activity (Drevets et al., 2008; Greicius et al., 2007), it has been suggested that ketamine-induced attenuation of sgACC activity may mediate ketamine's antidepressant effect.

Despite the establishment of differential time courses of ketamine-induced changes in the BOLD signal across the brain, subsequent phMRI studies have focused on a ketamine 'signal model', a single regressor used to quantify the ketamine BOLD response, modelled by a gamma variate $(\mathrm{GV})$ response function (De Simoni et al., 2013; Doyle et al., 2013; Javitt et al., 2018; Stone et al., 2015). Modelling the neural effect of ketamine with a single time course is an overly simplistic conceptualisation of brain dynamics, reliant on the assumption that a systemic average is representative of regional responses to ketamine. The ketamine electroencephalography (EEG) and magnetoencephalography (MEG) literature demonstrates the existence of multiple time courses of neural oscillatory activity with distinctive spatial topographies (Kochs et al., 1996; Lee et al., 2013; Muthukumaraswamy et al., 2015), suggesting that modelling BOLD changes with a single regressor cannot capture the full spectrum of ketamine-induced phMRI changes.

Electrophysiological techniques have a distinct advantage over techniques using the BOLD response in that they are a direct measure of neural activity. Ketamine stimulates the sympathetic nervous system, resulting in significant increases in heart rate and blood pressure (Liebe et al., 2017). Due to its indirect nature, the BOLD signal may be more susceptible to contamination by these physiological effects, as vascular changes modulate the neurovascular coupling characteristics responsible for generating the signal. To date, the phMRI literature has not considered the influence of ketamine-induced physiological changes on the phMRI response. Thus, the aim of this study was to further investigate ketamine-induced alterations in neural activity using conventional phMRI analysis techniques, applied to fMRI data collected simultaneously with EEG and physiological data. Simultaneous EEG/ fMRI offers the ability to discern the temporal and spatial dynamics of neural oscillatory changes as measured by EEG and correlate them with concurrent changes in the BOLD signal.

\section{Materials and methods}

\section{Participants}

In total, 30 healthy males were recruited as part of a larger study involving several simultaneous EEG/fMRI scan protocols.
Only the data from resting-state scans are presented here. Two participants' data could not be used due to interruption of the resting-state scan following the drug infusion, and four participants' data could not be used due to excessive movement affecting both the EEG and fMRI data. Therefore, the data of 24 participants are reported here (mean age 28.2 years; $\mathrm{SD}=6.4$; range 19-37). Participants were psychologically healthy with no prior history of mental disorders, and were screened for current depressive symptoms with the Beck Depression Inventory II (BDI-II) (Beck et al., 1996) prior to commencing the study. Those with a BDI-II score of 13 or higher (less than 13 indicates minimal depression) were excluded from the study. Participants were physically healthy, were not taking any central nervous system medications and were screened for current recreational drug use. Informed written consent was obtained from all participants. The study was approved by a local ethics committee.

\section{Procedure}

The experiment was a single-blind, placebo-controlled, randomised, three-way crossover design using ketamine, midazolam and placebo. Only ketamine data are presented here. Data were collected over three sessions, each separated by a minimum of 48 hours to allow a sufficient washout period. Racemic ketamine was administered as a $0.25 \mathrm{mg} / \mathrm{kg}$ bolus followed by a $0.25 \mathrm{mg} /$ $\mathrm{kg} / \mathrm{hr}$ infusion, controlled by an infusion pump (Alaris PK Syringe Pump, BD, USA). Drug infusion commenced 7 minutes into a 16-minute resting-state scan. Participants were instructed to keep their eyes open and fixated on a cross on a projection screen.

\section{Data acquisition}

EEG. EEG data were continuously recorded from 64 channels using the Standard BrainCap MR and BrainAmp MR Plus amplifiers (Brain Products, Munich, Germany). The EEG was acquired using the manufacturer standard cap layout, with the ground electrode located at $\mathrm{AFz}$, reference electrode at $\mathrm{FCz}$, and a dropdown electrode attached centrally to the participant's back for recording of electrocardiography. A Syncbox device (Brain Products $\mathrm{GmbH}$, Munich, Germany) was used to synchronise the EEG recording to the MR scanner clock. Impedances were typically kept below $10 \mathrm{k} \Omega$. EEG data were recorded with BrainVision Recorder software (Brain Products GmbH, Munich, Germany) and sampled at $5 \mathrm{kHz}$ with a resolution of $0.5 \mu \mathrm{V}$, range of $\pm 16.384 \mu \mathrm{V}$ and a bandwidth of 0.1 to $250 \mathrm{~Hz}$. The amplifier system was positioned on a sled behind the participant within the scanner bore to reduce cable length. The cables connecting the cap and the amplifiers were fixed with adhesive tape to prevent any additional movement-related artefacts.

Prior to entering the MR scanner, individual electrode positions were recorded using an ultrasound digitisation device (Zebris, Tubingen, Germany) for later source localisation and coregistration. Contrast markers (Vitamin E capsules) were attached to electrode positions $\mathrm{Cz}, \mathrm{F} 5, \mathrm{CP} 5$ and FC6.

fMRI. Imaging was performed using a 3T MR scanner (Siemens Skyra, Erlangen, Germany) with a 20-channel head coil. During fMRI scanning, 437 whole-brain image volumes, consisting of 30 interleaved axial slices each, were continuously acquired 
using a standard $\mathrm{T} 2 *$-weighted echo-planar imaging sequence (repetition time $(\mathrm{TR})=2200 \mathrm{~ms}$; echo time $(\mathrm{TE})=27.0 \mathrm{~ms}$; flip angle $=79^{\circ}$; field of view $(\mathrm{FOV})=215 \mathrm{~mm}^{2}$; voxel size $=3 \times$ $3 \times 3 \mathrm{~mm}$; slice gap $=1 \mathrm{~mm}$ ). In each session, prior to the removal of the EEG cap and its attached contrast markers, a lowresolution T1-weighted magnetisation-prepared rapid gradient echo (MPRAGE) image was acquired to capture electrode positions $\left(\mathrm{TR}=1900 \mathrm{~ms} ; \mathrm{TE}=3.21 \mathrm{~ms}\right.$, flip angle $=9^{\circ}$; FOV $=256 \mathrm{~mm}^{2} ; 96$ slices; voxel size $=1.3 \times 1.3 \times 2 \mathrm{~mm}$ )

In one of the three sessions for each participant, a high resolution T1-weighted MPRAGE image was acquired following the removal of the EEG electrode cap to avoid structural distortions (Klein et al., 2015) $(\mathrm{TR}=2100 \mathrm{~ms}$; $\mathrm{TE}=3.42 \mathrm{~ms}$; flip angle $=$ $9^{\circ} ; \mathrm{FOV}=256 \mathrm{~mm}^{2} ; 192$ slices; voxel size $=1 \times 1 \times 1 \mathrm{~mm}$ ).

Physiological data. During scanning, each participant's pulse was recorded via a probe over their left index finger and respiration rate was recorded and registered using a flexible pressure belt fitted around the upper abdomen (Biopac Systems Inc., California, USA). Participants were fitted with a nasal cannula to measure end-tidal oxygen and carbon dioxide levels. These measurements were all recorded with a Biopac MP150 system (California, USA). A blood pressure cuff was fitted around the right arm for periodic measurement of blood pressure between scans. For additional safety monitoring, a pulse oximeter on the left middle finger measured heart rate and blood oxygen saturation (Nonin, USA).

\section{EEG data analysis}

Preprocessing. The scanner-induced gradient artefact, caused by the fast switching of the MR gradients during fMRI acquisition, was reduced by realignment parameter-informed template subtraction (Moosmann et al., 2009), a modified version of standard template artefact subtraction (Allen et al., 2000). The data were subsequently down-sampled to $500 \mathrm{~Hz}$ and bandpass filtered to between 0 and $100 \mathrm{~Hz}$.

The ballistocardiogram artefact, caused by the pulsatile motion of blood in the head, was corrected using an automated approach combining independent components analysis (ICA) with single value decomposition (SVD) to remove and/or filter components from the data that share high levels of mutual information with the cardiac trace (Liu et al., 2012).

The raw EEG was visually inspected for manual identification of remaining motion or muscle artefact, which were consequently removed. Subsequently, ICA was used to manually identify common EEG artefacts, such as ocular artefacts, for removal. The data were epoched into $2200 \mathrm{~ms}$ segments, corresponding to volumes collected with $\mathrm{fMRI}$, and then referenced to the common average reference, recovering activity at electrode FCz.

Spectral analysis. For source-localisation, individual electrode positions were co-registered to the structural MRI of each participant. For source modelling, three-shell boundary element models were constructed for each session using brain, skull and scalp layers. Global covariance matrices were generated for the data, which were filtered into seven frequency bands using a fourth order bidirectional Butterworth filter: delta $(1-4 \mathrm{~Hz})$, theta
(4-8 Hz), alpha (8-13 Hz), low beta (15-26 Hz), high beta (28$40 \mathrm{~Hz})$, low gamma $(42-53 \mathrm{~Hz})$ and high gamma $(55-67 \mathrm{~Hz})$. These frequencies were chosen to avoid the slice artefact frequency and its harmonics. Linearly constrained minimum variance beamforming (Van Veen et al., 1997) was applied using 8 mm resolution grids warped to the template head-model provided with FieldTrip (Oostenveld et al., 2011) to generate spatial filters for each voxel. Beamformer maps measuring percentage change in source power for pre- versus post-ketamine were computed for each individual. At the group level, two-tailed, one-sample $t$ tests were computed using the FMRIB Software Library's (FSL's) randomise (Winkler et al., 2014) to correct for multiple comparisons using threshold-free cluster enhancement (Smith and Nichols, 2009) and a defined significance threshold of $p<0.05$.

To generate EEG regressors for each frequency band for each individual, the coordinate corresponding to the peak power intensity was selected and used to construct a virtual sensor from which the power time series was extracted. The time series was thresholded to remove values exceeding five standard deviations from the mean. These values and those missing due to removal during data cleaning were linearly interpolated. Finally, a lowpass Butterworth IIR filter $(0.0167 \mathrm{~Hz})$ was applied to the power time series to focus on slow power changes, equivalent to the phMRI response.

\section{fMRI data analysis}

Preprocessing. fMRI image pre-processing was performed using tools from FSL (Jenkinson et al., 2012; Smith et al., 2004; Woolrich et al., 2009). The following pre-statistics processing was applied: motion correction using MCFLIRT (Jenkinson et al., 2002), slice timing correction using Fourier-space timeseries phase-shifting, non-brain removal using BET (Smith, 2002), and grand-mean intensity normalisation. Registration to high resolution structural images was carried out using FLIRT (Jenkinson et al., 2002; Jenkinson and Smith, 2001). Registration from high resolution structural space to MNI standard space was further refined using FNIRT (Andersson et al., 2007). Images were spatially smoothed using a Gaussian kernel of $8 \mathrm{~mm}$ FWHM. No temporal filtering was applied for the pseudo-block phMRI analysis. Images were temporally filtered with a high pass filter of $960 \mathrm{~s}(0.001 \mathrm{~Hz})$ for the ketamine signal model and EEGinformed phMRI analyses.

\section{phMRI data analysis}

To establish the validity of our fMRI data collected during a simultaneous EEG recording, we replicated aspects of two previous ketamine phMRI studies: a pseudo-block analysis method (Deakin et al., 2008) and a method utilising a ketamine 'signal model' (De Simoni et al., 2013). This was followed by a novel EEG-informed phMRI analysis.

Pseudo-block phMRI analysis. We replicated the pseudo-block analysis method of Deakin et al. (2008) at the single subject level. Using FSL's FEAT, a regression analysis was computed using the general linear model (GLM) for each participant. The 7 minutes of pre-infusion data were treated as a baseline block and each subsequent minute of the ketamine infusion was treated as 
an independent test block. The baseline block and nine 1-minute ketamine infusion blocks were entered into a design matrix as separate columns. At the group level, a one-way analysis of variance was calculated to assess the group average effect of ketamine. $F$-statistic images were thresholded using clusters determined by $Z>2.3$ and a familywise error (FWE) corrected cluster significance threshold of $p=0.05$ was applied (Worsley, 2001).

This analysis was repeated with the inclusion of various nuisance regressors at the single subject level to understand their influence on the BOLD signal changes occurring with ketamine infusion. The first repetition included the addition of the sixstandard motion regressors. The second repetition included physiological correction by the addition of an end-tidal $\mathrm{CO}_{2}$ regressor and its temporal derivative, and 34 slice-wise physiological regressors created using FSL's Physiological Noise Modelling (PNM) toolbox (Brooks et al., 2008). These PNM regressors included those for the cardiac and respiratory cycles (Glover et al., 2000), respiratory volume over time (Chang and Glover, 2009) and heart rate (Chang et al., 2009). Finally, the third repetition included all regressors from both repetition one and two, namely, both motion and physiological regressors.

To test whether increasing model complexity modified the GLM fits, we used the technique of Bright and Murphy (2015) to create simulated motion regressors. The true motion regressors were repeatedly phase randomised until the temporal correlation with the true regressors was $r<0.1$, before being entered into the model at the single subject level.

Ketamine signal model phMRI analysis. We replicated the six design matrices (models) defined by De Simoni et al. (2013) in which the ketamine phMRI response was modelled with a GV 'signal model' regressor. A shape capturer regressor was defined to model variation in the peak time and shape of the signal model regressor. Motion was modelled by inclusion of all six standard motion parameters or by the inclusion of the first SVD component obtained from the six standard motion parameters following normalisation. A linear drift regressor was included in all models. The design matrices evaluated were:

Model 1: ketamine signal model, linear drift;

Model 2: ketamine signal model, linear drift, shape capturer;

Model 3: ketamine signal model, linear drift, first SVD component of motion regressors;

Model 4: ketamine signal model, linear drift, shape capturer, first SVD component of motion regressors;

Model 5: ketamine signal model, linear drift, six standard motion regressors;

Model 6: ketamine signal model, linear drift, shape capturer, six standard motion regressors.

At the group level for each model, a group average was calculated. $Z$ statistic images were thresholded using clusters determined by $Z>2.3$ and a FWE-corrected cluster significance threshold of $p=0.05$ was applied (Worsley, 2001).

As De Simoni et al. (2013) chose Model 3 for further description of the ketamine phMRI response, we used Model 3 to investigate the effect of physiological correction. To the Model 3 design matrix, we added physiological regressors, as described above, at the single subject level.

To test whether increasing model complexity with the addition of physiological noise regressors modified the GLM fits, we performed an additional analysis in which we created simulated physiological noise regressors through phase randomisation (as described previously) and these regressors were entered into the model at the single subject level.

EEG-informed phMRI analysis. For the novel EEG-informed phMRI analysis, ketamine-induced changes in activation were determined for each EEG frequency band at the single subject level. Using FSL's FEAT, a regression analysis was computed using the GLM for each participant. The regressors included the power time course for the frequency band of interest, convolved with a double gamma haemodynamic response function and its temporal derivative, and six standard motion regressors. At the group level, an average was calculated. $Z$ statistic images were thresholded using clusters determined by $Z>2.3$ and an FWEcorrected cluster significance threshold of $p=0.05$ was applied (Worsley, 2001).

This analysis was then repeated with physiological correction, with physiological regressors described above added to the GLM at the single subject level.

\section{Results}

\section{Physiological changes}

Consistent with known physiological effects, ketamine caused increased heart rate, blood pressure, and respiratory volume per unit time, but no change in end-tidal $\mathrm{CO}_{2}$ (Figure 1). The time course of the ketamine-induced increase in heart rate was significantly correlated with the ketamine GV signal model ( $r=0.96$, $p<0.0001)$.

\section{Pseudo-block phMRI analysis}

Replication of the pseudo-block analysis used by Deakin et al. (2008) produced comparable results, with increases in BOLD signal in the ACC, paracingulate gyrus, frontal pole, supplementary motor cortex, precuneus, lateral occipital cortex, occipital pole and thalamus and decreases in the sgACC, medial frontal cortex and the right postcentral gyrus (Figure 2(a)). BOLD signal changes from three regions of interest displayed different time courses.

With the addition of the six standard motion regressors to the original analysis, the BOLD signal increases remained largely unchanged from the original analysis. However, the decreases in BOLD signal in the sgACC, medial frontal cortex and right postcentral gyrus were no longer detected (Figure 2(b)). When this analysis was repeated with simulated motion regressors, BOLD signal decreases in the sgACC, medial frontal cortex, and right postcentral gyrus were detected (Figure S1).

With the addition of physiological regressors to the original analysis, decreases in BOLD signal in the sgACC, medial frontal cortex and the right postcentral gyrus were detected (Figure 2(c)), while the increases in BOLD signal became less widespread, particularly in the supplementary motor cortex and precuneus. The 


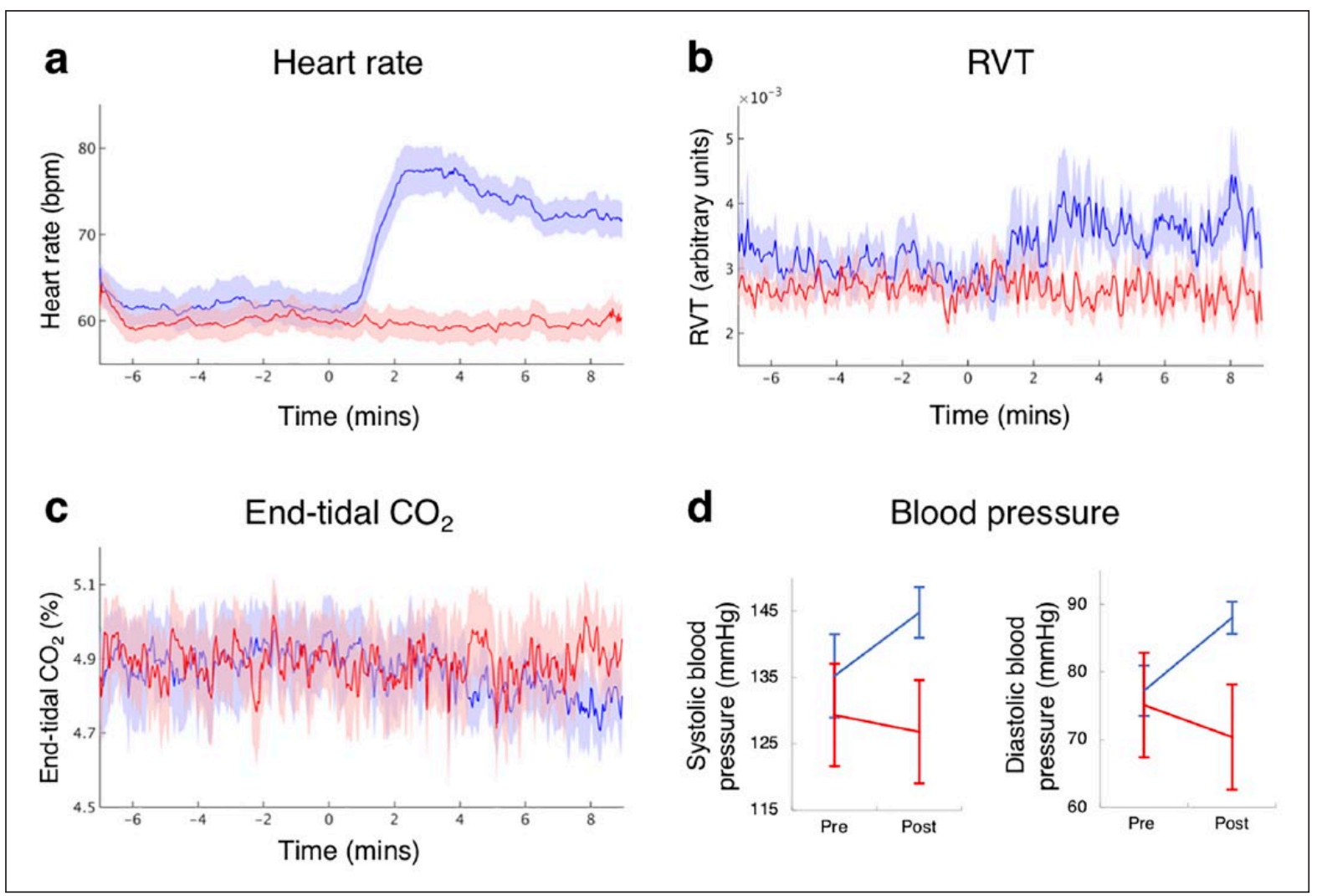

Figure 1. Basic physiological measures before and during ketamine infusion or placebo. Blue is ketamine; red is placebo. Infusion started at time 0 . Error bars/shading are the standard error of the mean. (a)-(b) As expected, participants demonstrated increases in heart rate $(t=9.48, p<0.001)$ and respiratory volume per unit time (RVT) $(t=2.65, p<0.02)$. (c)-(d) Participants did not show a change in end-tidal $\mathrm{CO}_{2}(t=-0.57, p=0.57)$ but did show increased systolic $(F(1,18)=11.39, p<0.004)$ and diastolic blood pressure $(F(1,18)=19.11, p<0.001)$.

addition of physiological regressors appeared to introduce spurious decreases in BOLD signal in small regions of white matter, similar to those seen in Figure 2(a). Approaches to physiological correction of pharmacological resting state fMRI data have been shown to produce false positives involving white matter (KhaliliMahani et al., 2013) but nevertheless provide important control for drug-induced physiological fluctuations in the BOLD signal.

The spurious decreases in the BOLD signal in the white matter were not present when both motion and physiological regressors were added to the original analysis, raising the possibility of an association of these effects with head movement. In this analysis, the BOLD signal decreased in the bilateral precentral and postcentral gyri (Figure 2(d)). Whereas this BOLD signal decrease appears similar to an edge effect, alternative views of the group map of this analysis show localisation of this effect along the precentral and postcentral gyri (Figure S2). No change in BOLD signal was detected in the SgACC or medial frontal cortex and the cortical increases in BOLD signal were less widespread, particularly in the frontal pole. However, there was a more extensive bilateral thalamic BOLD signal increase.

\section{Ketamine signal model phMRI analysis}

Replication of the six ketamine signal model design matrices defined by De Simoni et al. (2013) produced comparable results (Figure S3). In all models, ketamine induced widespread increases in BOLD signal across the majority of the cortex. Furthermore, all models detected a bilateral signal decrease in the sgACC, despite De Simoni et al. (2013) only detecting this change with Model 3. Our detection of this change with all models is potentially due to the increased power provided by our substantially larger sample size ( $n=24$ vs $n=10)$. A BOLD signal decrease in the left temporal pole was detected with Models 1, 3, 5 and 6.

Further analysis using Model 3 showed the signal decreases in the bilateral sgACC and left temporal pole were no longer detected when physiological noise correction was included in the model (Figure 3). When this analysis was repeated with simulated physiological noise regressors, BOLD signal decreases in the bilateral sgACC and left temporal pole were detected (Figure S4).

\section{EEG spectral changes}

Ketamine-induced EEG spectral changes were computed in the source space (Figure 4(a)). Source power in the theta, high beta, low gamma and high gamma frequency bands decreased whereas increases in source power were observed in the delta, alpha and low beta frequency bands. The spectral changes and their localisations are entirely consistent with ketamine-induced source power changes measured with MEG using a similar subanaesthetic dose of ketamine (Muthukumaraswamy et al., 2015). 


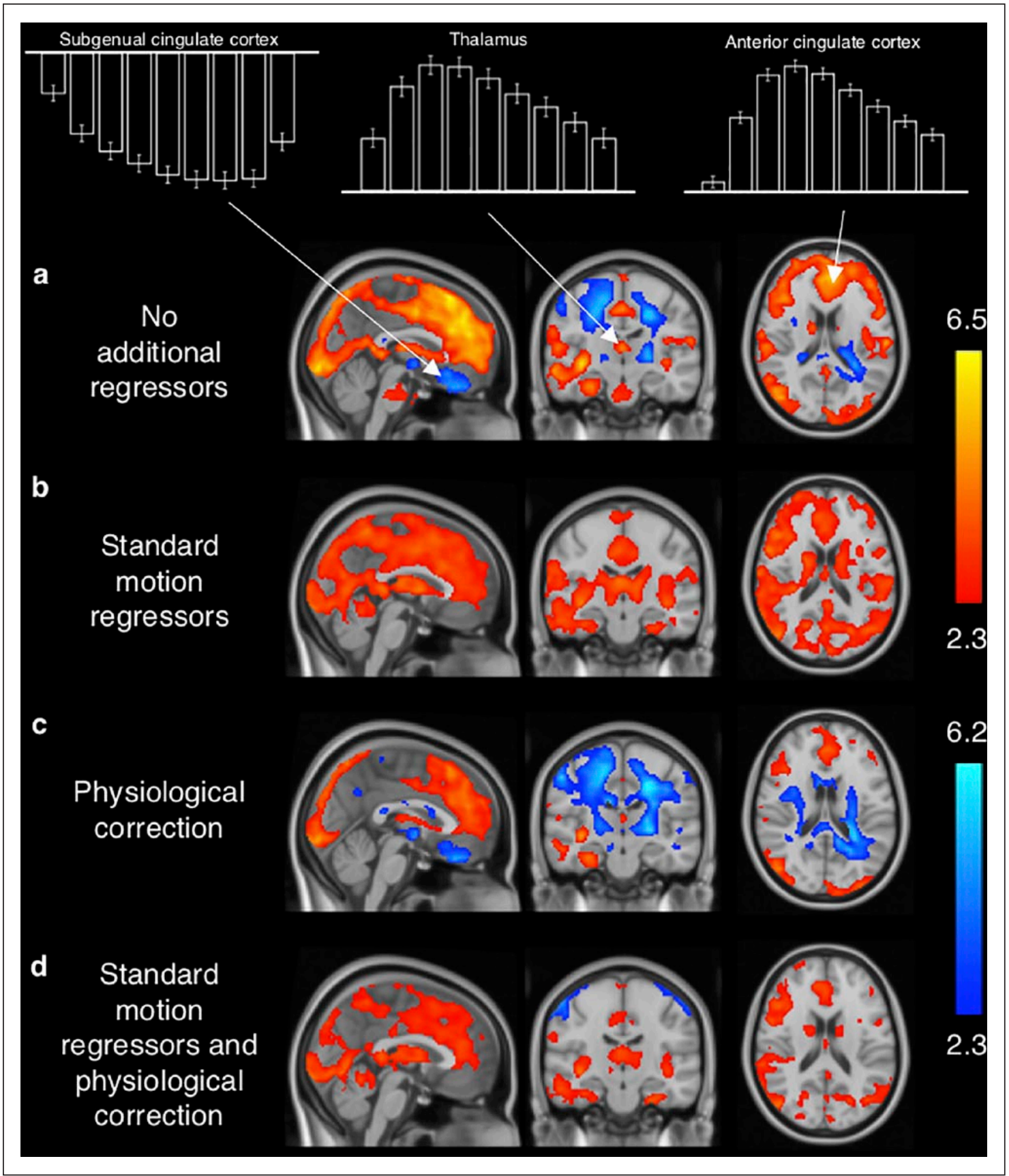

Figure 2. Group maps showing the locations of significant ( $F$-ratio maps, $p<0.05$, familywise error (FWE) cluster corrected) effects of ketamine on the blood-oxygenation-level-dependent (BOLD) signal during the first 9 minutes of ketamine infusion. Although these are $F$-ratio maps, for visual display purposes, areas that showed decreased BOLD responses post-hoc are coloured blue. A grey matter mask was applied to all maps. The histograms show the time series of the BOLD signal change in 1-minute increments from three selected regions showing a significant effect of ketamine. Error bars represent $90 \%$ confidence intervals. (a) Replication of pseudoblock analysis technique of Deakin et al. (2008), in which the general linear model (GLM) did not include motion regressors. (b) Pseudoblock analysis with the addition of the six standard motion parameters as regressors in the GLM. (c) Pseudoblock analysis with physiological correction by addition of an end-tidal $\mathrm{CO}_{2}$ regressor and its temporal derivative, and 34 slice-wise physiological regressors created with the PNM toolbox to the GLM. (d) Pseudoblock analysis with the addition of the six standard motion parameters to the GLM and physiological correction as described in (c).

The time courses of these source power changes showed variation in temporal profile across the different frequency bands (Figure 4(b)). The largest increases, with the shortest onset latency, occurred in frontal theta and posterior high gamma power. Smaller increases in power that were slower to reach a maximum were observed for posterior low gamma and high beta. 


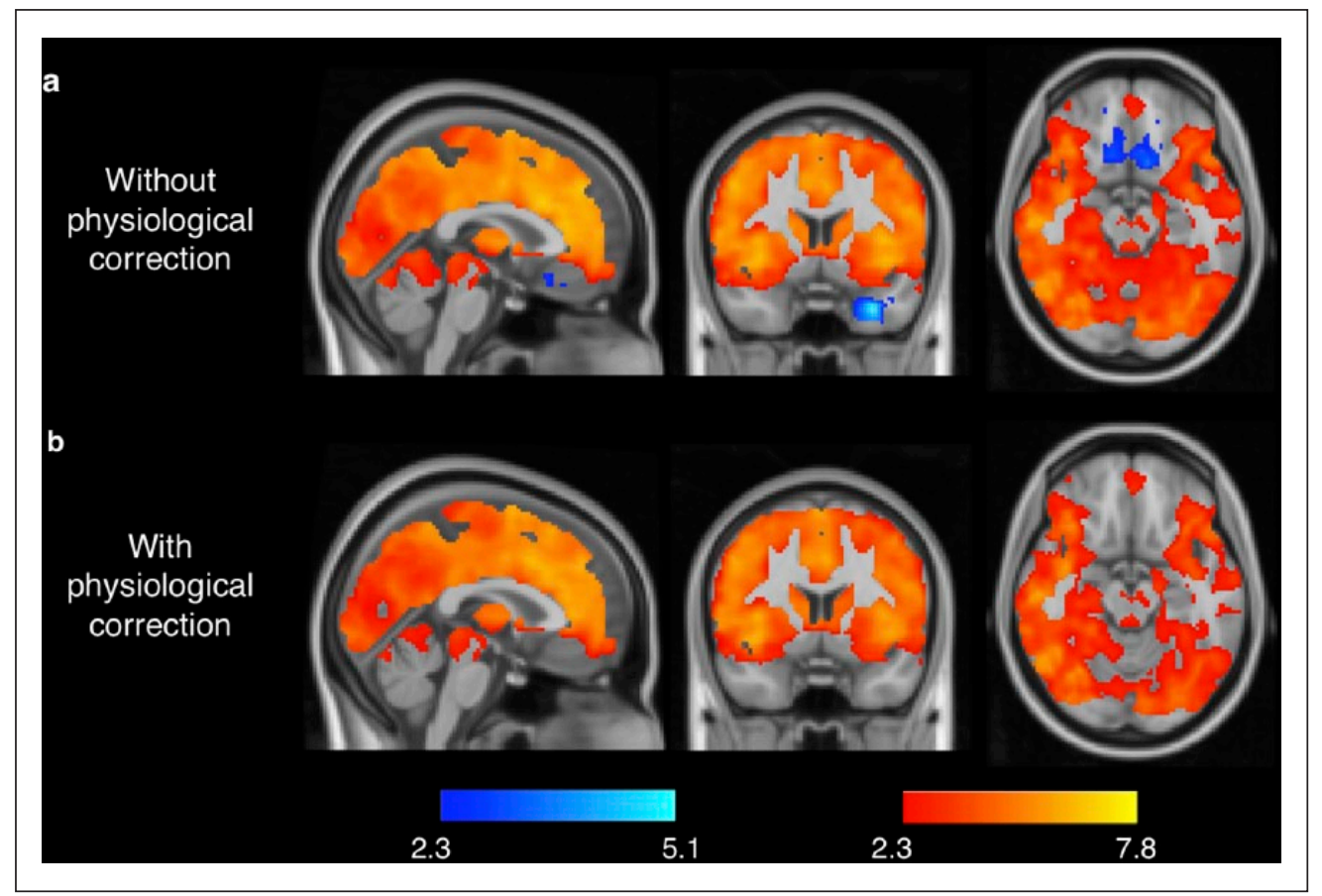

Figure 3. Group maps of modelled ketamine blood-oxygenation-level-dependent (BOLD) response for Model 3 of De Simoni et al. (2013) showing significant effects of ketamine ( $p<0.05$, familywise error (FWE) cluster corrected). The Model 3 design matrix included the gamma variate ketamine signal model, the first single value decomposition (SVD) component of individual motion traces, and a linear drift term. A grey matter mask was applied to both maps. (a) Replication of Model 3 of De Simoni et al. (2013) in which the general linear model (GLM) did not include any physiological correction. (b) As in (a) but with addition of an end-tidal $\mathrm{CO}_{2}$ regressor and its temporal derivative, and 34 slice-wise physiological regressors created with the Physiological Noise Modelling (PNM) toolbox to the design matrix.

The largest decrease in power occurred for posterior alpha, and smaller, slower decreases in power were seen in the posterior delta and low beta frequency bands.

\section{EEG-informed phMRI analysis}

The BOLD signal was temporally correlated with EEG source power in specific frequency bands in relatively distinct spatial areas for both the data that was physiologically corrected (Figure 5) and that which was not (Figure S5). In general, physiological correction increased the spatial spread of negative correlations between the BOLD signal and EEG source power, whereas it decreased the spatial spread of positive correlations.

Frontal theta source power positively correlated with the BOLD signal from the frontal pole, ACC, paracingulate gyrus, inferior frontal gyrus, insula, supplementary motor area, precentral gyrus, precuneus, lingual gyrus and the lateral occipital cortex. For the high beta and high gamma frequency bands there were positive correlations with the BOLD signal, although to a considerably smaller spatial extent than the other frequency bands. Posterior high beta source power positively correlated with BOLD signal from the inferior frontal regions of the ACC extending into the sgACC, the right supplementary motor cortex, and the left central opercular cortex, precentral gyrus and inferior frontal gyrus. There were significant correlations between BOLD signal and high gamma in the precentral gyrus, supplementary motor cortex and adjacent superior regions of the ACC. There were no significant correlations between the BOLD signal and low gamma source power.

Both posterior delta and low beta source power were negatively correlated with BOLD signal from areas including the bilateral thalamus, PCC, precuneus and the left interior frontal gyrus. Additionally, further negative correlations between delta source power and BOLD signal were seen in the ACC and paracingulate gyrus. Posterior alpha source power was negatively correlated with the BOLD signal from the anterior and posterior cingulate cortices, paracingulate gyrus, inferior frontal gyrus, insula, thalamus, precuneus, superior parietal lobule, occipital pole and the left lateral occipital cortex.

\section{Discussion}

We used simultaneous EEG/fMRI to assess the effect of a subanaesthetic dose of ketamine on neural activity, demonstrating that ketamine causes widespread cortical increases in the BOLD signal and that this correlates with temporally diverse EEG power changes in various frequency bands. To our knowledge, this is the first study to use simultaneous EEG/fMRI to investigate the phMRI response. Despite the relatively hostile EEG recording environment and associated artefacts (Fellner et al., 2016), the pattern and localisation of EEG spectral responses are strikingly similar to previous MEG studies (Muthukumaraswamy et al., 2015; Rivolta et al., 2015). Similarly, the phMRI results, indexing ketamine-related changes in the BOLD signal, are consistent 


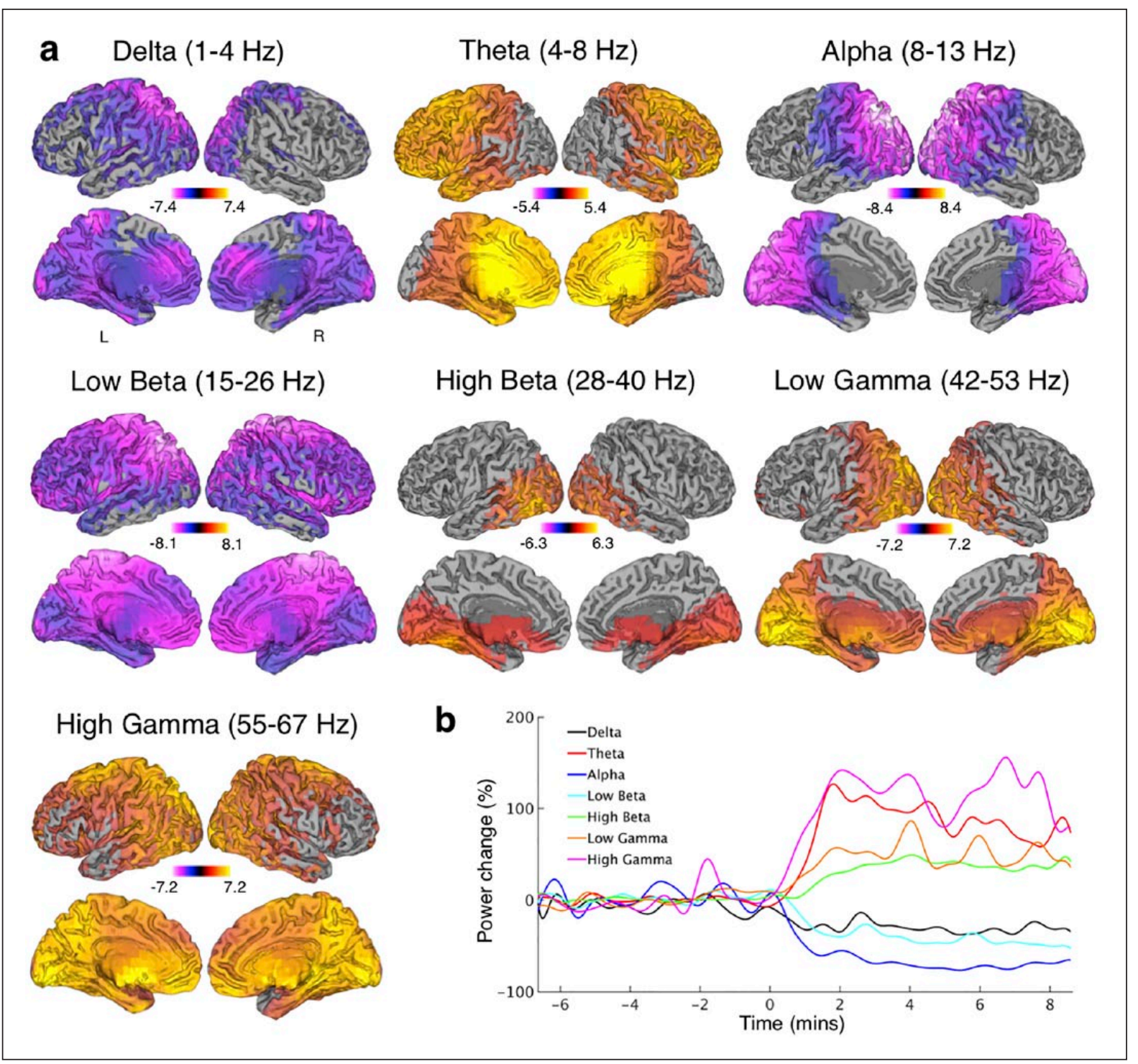

Figure 4. (a) Statistical parametric maps showing the locations of significant $(p<0.025$, corrected) changes in oscillatory source power in seven frequency bands that span from 1 to $67 \mathrm{~Hz}$. Contrasts of spectral power represent the difference of ketamine after and before infusion. Warm colours represent increases in source power and cool colours represent decreases. (b) Time courses of band-limited virtual sensor power for seven frequency bands that span from 1 to $67 \mathrm{~Hz}$, computed as a percentage change from the pre-infusion time period $(-7-0 \mathrm{~min})$. Delta $=1-4 \mathrm{~Hz}$, theta $=4-8 \mathrm{~Hz}$, alpha $=8-13 \mathrm{~Hz}$, low beta $=15-26 \mathrm{~Hz}$, high beta $=28-40 \mathrm{~Hz}$, low gamma $=42-53 \mathrm{~Hz}$, high gamma $=55-67 \mathrm{~Hz}$.

with previous analyses in the ketamine phMRI literature (Deakin et al., 2008; De Simoni et al., 2013). Critically, however, our more extensive analyses indicate the importance of motion and physiological nuisance regressors in the analysis of the effects of ketamine. We replicated the earlier findings of two previous studies (Deakin et al., 2008; De Simoni et al., 2013) that showed widespread increases in cortical BOLD signal and a reduction in BOLD signal in the sgACC. However, the decrease in BOLD signal in the sgACC was attenuated by the addition of motion and physiological nuisance regressors when modelling the data. The repetition of these analyses with simulated motion and physiological noise regressors confirmed the attenuation of the BOLD signal change in the sgACC was not the result of a reduced variation in the data leading to reduced sensitivity to detect changes due to the addition of more regressors to the model.
The acute neural effects of low-dose ketamine are thought to involve a surge of glutamate release due to the disinhibition of cortical pyramidal neurons (Homayoun and Moghaddam, 2007). Previous studies have utilised pre-treatment with psychoactive drugs known to reduce cortical glutamate release, to attempt to attenuate the BOLD response to ketamine. Pre-treatment with lamotrigine, a voltage-gated sodium channel blocker, attenuates the BOLD signal increases associated with ketamine but does not affect the decrease in the BOLD signal in the sgACC (Deakin et al., 2008; Doyle et al., 2013). However, pre-treatment with the atypical antipsychotic risperidone attenuates both the BOLD increases and the decrease in the sgACC (Doyle et al., 2013). It is plausible that pre-treatment with risperidone may reduce the cardiovascular changes produced by ketamine, through modulation of ketamine's effects on the sympathetic nervous system via 


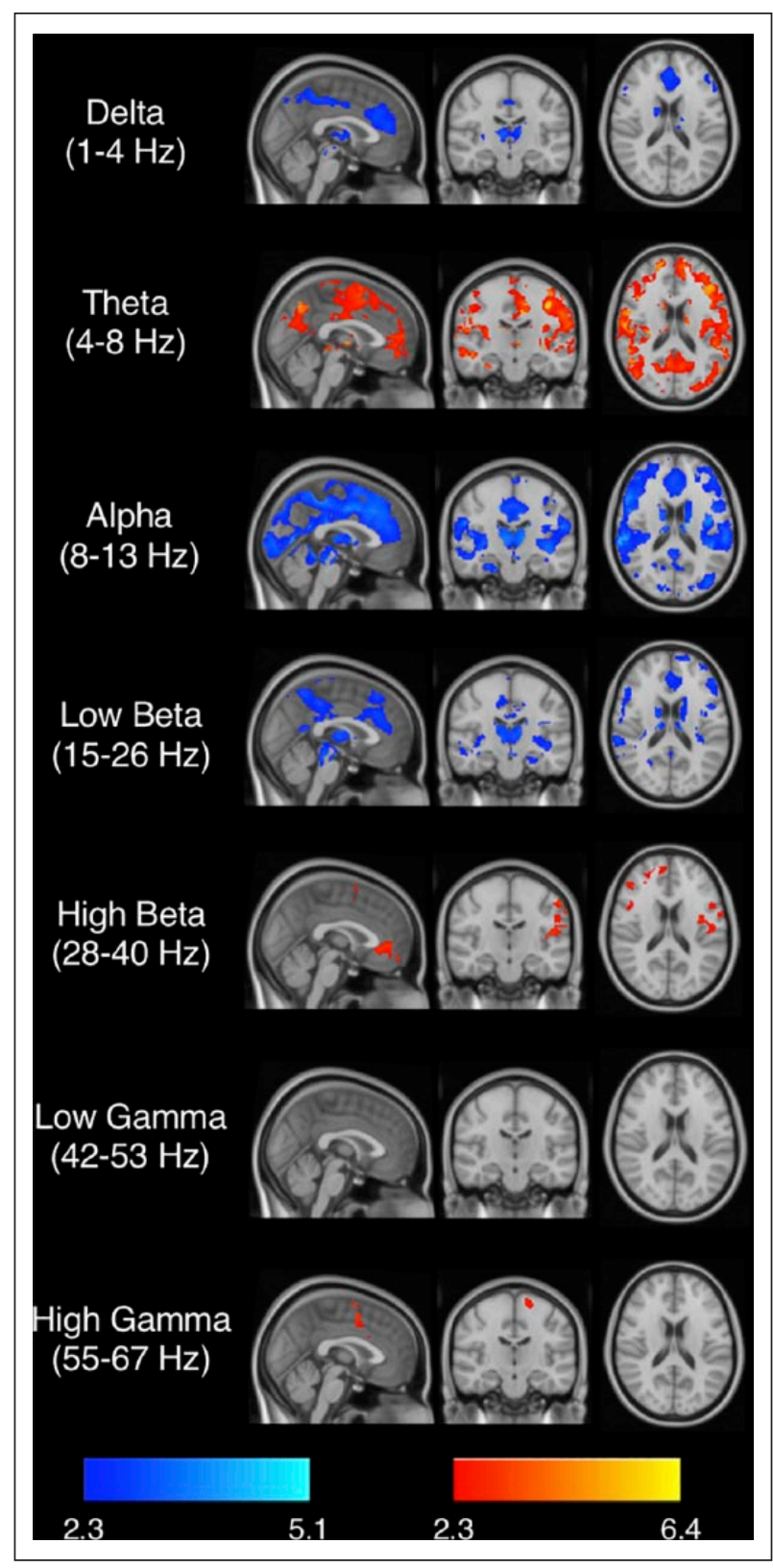

Figure 5. Group maps of modelled ketamine blood-oxygenation-leveldependent (BOLD) response for time courses of band-limited virtual sensor electroencephalography (EEG) power for seven frequency bands that span from 1 to $67 \mathrm{~Hz}$, showing significant effects of ketamine ( $p<0.05$, familywise error (FWE) cluster corrected). Physiological correction was applied in this analysis; the design matrix included an end-tidal $\mathrm{CO}_{2}$ regressor and its temporal derivative, and 34 slice-wise physiological regressors created with the Physiological Noise Modelling (PNM) toolbox. A grey matter mask was applied to all maps.

risperidone's action as an antagonist at alpha-1 and alpha-2 adrenergic receptors (He and Richardson, 1995). This is consistent with our observation that no change in BOLD signal is detected in the sgACC when motion and/or physiological noise regressors are included in the model, suggesting the signal change observed in the sgACC is the result of physiological noise. This region lies in close proximity to the anterior cerebral artery (ACA), potentially increasing its sensitivity to physiological noise, in particular cardiac pulsatility, which can produce cardiac-related bulk motion of the surrounding brain tissue (Dagli et al., 1999) and is more highly localised than respiratory noise (Glover et al., 2000). Recently developed phase-based amplified MRI has demonstrated tissue deformation in the sgACC and adjacent frontal brain regions with the pulsatile motion of the ACA (Terem et al., 2018).

A phMRI study in individuals with MDD did not replicate the decrease in BOLD signal in the sgACC associated with ketamine infusion (Downey et al., 2016). The increase in BOLD signal that occurred in this region was attributed to the method of ketamine administration (slow infusion without an initial bolus). However, given the study was in a clinical population and failed to replicate the widely reproduced antidepressant effect of ketamine (see Abdallah et al. (2015) for review), this finding is difficult to interpret.

Our EEG-informed phMRI analysis provides evidence for distinct temporal time courses of neural response to ketamine and shows that modelling the phMRI response with a single temporal model does not capture the full spectrum of neuronal dynamics. Similar to previous studies (Kochs et al., 1996; Lee et al., 2013; Muthukumaraswamy et al., 2015), the EEG power time series in different frequency bands had distinct temporal characteristics and here we show these temporal dynamics are correlated with BOLD signal changes in relatively distinct spatial areas, particularly when physiological nuisance regressors are included in the analysis model. The widespread cortical increase in BOLD signal typically seen in the literature positively correlated with frontal theta power, which followed a similar time course to the ketamine signal model. The use of EEG power time series, as opposed to a ketamine signal model, offers the distinct advantage of using regressors that are direct measures of neural activity. Furthermore, the regressors are individualised, corresponding to the individual's neural activity in a particular frequency band, rather than being generic like the signal model. Of particular interest is the observation that no EEG power time series in any of the frequency bands correlated with a decrease in BOLD signal in the sgACC. Our analysis only included the primary source within each EEG frequency band and it is possible there are correlations between secondary EEG power sources and the BOLD signal. However, it is difficult to characterise secondary sources and achieve correspondence across participants, and our exploratory analyses suggest these secondary sources often display similar low-frequency temporal dynamics to the primary sources. Furthermore, a principle components analysis of the EEG data showed the data had a very low rank $(\sim 50)$ and, on average, there was only one principle component explaining more than $5 \%$ of the variance within each frequency band for each individual participant (Supplementary Table 1). As such, we believe this justifies the modelling of the phMRI response with a single individualised time course for each EEG frequency band.

In summary, this study highlights the influence of physiological noise on the ketamine phMRI response, particularly in the sgACC. Furthermore, correlations between the BOLD signal and the EEG power time series in seven different frequency bands demonstrate the presence of multiple time courses of ketamine-induced neural activity. This indicates that the full spectrum of neuronal dynamics associated with ketamine cannot be captured by a single temporal 
model and we therefore recommend the use of EEG-based regressors, which directly reflect neural activity and can serve as an alternative approach to the ketamine signal model.

\section{Declaration of conflicting interest}

The author(s) declared no potential conflicts of interest with respect to the research, authorship, and/or publication of this article.

\section{Funding}

The author(s) disclosed receipt of the following financial support for the research, authorship, and/or publication of this article: This work was funded by F Hoffman La Roche Ltd.

\section{Supplementary material}

Supplementary material for this article is available online.

\section{ORCID iD}

Rebecca McMillan (iD) https://orcid.org/0000-0002-4811-5187

\section{References}

Abdallah CG, Averill LA and Krystal JH (2015) Ketamine as a promising prototype for a new generation of rapid-acting antidepressants. Ann N Y Acad Sci 1344: 66-77.

Allen PJ, Josephs O and Turner R (2000) A method for removing imaging artifact from continuous EEG recorded during functional MRI. Neuroimage 12: 230-239.

Andersson JL, Jenkinson M and Smith S (2007) Non-Linear Registration, Aka Spatial Normalisation FMRIB Technical Report TR07JA2. 28 June. Oxford: FMRIB Analysis Group of the University of Oxford, p. 2.

Beck A, Steer R and Brown G (1996) Manual for the Beck Depression Inventory-II San Antonio. San Antonio: Psychological Corporation.

Bright MG and Murphy K (2015) Is fMRI "noise" really noise? Resting state nuisance regressors remove variance with network structure. Neuroimage 114: 158-169.

Brooks JC, Beckmann CF, Miller KL, et al. (2008) Physiological noise modelling for spinal functional magnetic resonance imaging studies. Neuroimage 39: 680-692.

Chang C, Cunningham JP and Glover GH (2009) Influence of heart rate on the BOLD signal: The cardiac response function. Neuroimage 44: $857-869$.

Chang C and Glover GH (2009) Relationship between respiration, endtidal $\mathrm{CO}_{2}$, and BOLD signals in resting-state fMRI. Neuroimage 47: 1381-1393.

Dagli MS, Ingeholm JE and Haxby JV (1999) Localization of cardiacinduced signal change in fMRI. Neuroimage 9: 407-415.

Deakin JW, Lees J, McKie S, et al. (2008) Glutamate and the neural basis of the subjective effects of ketamine: A pharmaco-magnetic resonance imaging study. Arch Gen Psychiatry 65: 154-164.

De Simoni S, Schwarz AJ, O'Daly OG, et al. (2013) Test-retest reliability of the BOLD pharmacological MRI response to ketamine in healthy volunteers. Neuroimage 64: 75-90.

Downey D, Dutta A, McKie S, et al. (2016) Comparing the actions of lanicemine and ketamine in depression: Key role of the anterior cingulate. Eur Neuropsychopharmacol 26: 994-1003.

Doyle O, De Simoni S, Schwarz A, et al. (2013) Quantifying the attenuation of the ketamine pharmacological magnetic resonance imaging response in humans: A validation using antipsychotic and glutamatergic agents. J Pharmacol Exper Ther 345: 151-160.

Drevets WC, Savitz J and Trimble M (2008) The subgenual anterior cingulate cortex in mood disorders. CNS Spectr 13: 663.
Fellner M-C, Volberg G, Mullinger KJ, et al. (2016) Spurious correlations in simultaneous EEG-fMRI driven by in-scanner movement. Neuroimage 133: 354-366.

Frohlich J and Van Horn JD (2014) Reviewing the ketamine model for schizophrenia. J Psychopharmacol 28: 287-302.

Glover GH, Li TQ and Ress D (2000) Image-based method for retrospective correction of physiological motion effects in fMRI: RETROICOR. Magn Reson Med 44: 162-167.

Greicius MD, Flores BH, Menon V, et al. (2007) Resting-state functional connectivity in major depression: Abnormally increased contributions from subgenual cingulate cortex and thalamus. Biol Psychiatry 62: 429-437.

$\mathrm{He} \mathrm{H}$ and Richardson J (1995) A pharmacological, pharmacokinetic and clinical overview of risperidone, a new antipsychotic that blocks serotonin 5- $\mathrm{HT}_{2}$ and dopamine $\mathrm{D}_{2}$ receptors. Int Clin Psychopharmacol 10: 19-30.

Höflich A, Hahn A, Küblböck M, et al. (2016) Ketamine-dependent neuronal activation in healthy volunteers. Brain Struct Funct 222: $1-10$.

Homayoun H and Moghaddam B (2007) NMDA receptor hypofunction produces opposite effects on prefrontal cortex interneurons and pyramidal neurons. J Neurosci 27: 11,496-11,500.

Javitt DC, Carter CS, Krystal JH, et al. (2018) Utility of imagingbased biomarkers for glutamate-targeted drug development in psychotic disorders: A randomized clinical trial. JAMA Psychiatry 75: 11-19.

Jenkinson M, Bannister P, Brady M, et al. (2002) Improved optimization for the robust and accurate linear registration and motion correction of brain images. Neuroimage 17: 825-841.

Jenkinson M, Beckmann CF, Behrens TE, et al. (2012) FSL. Neuroimage 62: 782-790.

Jenkinson M and Smith S (2001) A global optimisation method for robust affine registration of brain images. Med Image Anal 5: 143-156.

Khalili-Mahani N, Chang C, van Osch MJ, et al. (2013) The impact of "physiological correction" on functional connectivity analysis of pharmacological resting state fMRI. Neuroimage 65: 499-510.

Klein C, Hänggi J, Luechinger R, et al. (2015) MRI with and without a high-density EEG cap: What makes the difference? Neuroimage 106: 189-197.

Kochs E, Scharein E, Mollenberg O, et al. (1996) Analgesic efficacy of low-dose ketamine. Somatosensory-evoked responses in relation to subjective pain ratings. J Am Soc Anesthesiol 85: 304-314.

Lee U, Ku S, Noh G, et al. (2013) Disruption of frontal-parietal communication by ketamine, propofol, and sevoflurane. J Am Soc Anesthesiol 118: 1264-1275.

Liebe T, Li S, Lord A, et al. (2017) Factors influencing the cardiovascular response to subanesthetic ketamine: A randomized, placebocontrolled trial. Int J Neuropsychopharmacol 20: 909-918.

Liu Z, de Zwart JA, van Gelderen P, et al. (2012) Statistical feature extraction for artifact removal from concurrent fMRI-EEG recordings. Neuroimage 59: 2073-2087.

Moosmann M, Schönfelder VH, Specht K, et al. (2009) Realignment parameter-informed artefact correction for simultaneous EEG-fMRI recordings. Neuroimage 45: 1144-1150.

Muthukumaraswamy SD, Shaw AD, Jackson LE, et al. (2015) Evidence that subanesthetic doses of ketamine cause sustained disruptions of NMDA and AMPA-mediated frontoparietal connectivity in humans. J Neurosci 35: 11694-11706.

Oostenveld R, Fries P, Maris E, et al. (2011) FieldTrip: Open source software for advanced analysis of MEG, EEG, and invasive electrophysiological data. Comput Intell Neurosci 2011: 1.

Rivolta D, Heidegger T, Scheller B, et al. (2015) Ketamine dysregulates the amplitude and connectivity of high-frequency oscillations in cortical-subcortical networks in humans: Evidence from resting-state magnetoencephalography-recordings. Schizophr Bull 41: $1105-1114$. 
Smith SM (2002) Fast robust automated brain extraction. Hum Brain Mapp 17: 143-155.

Smith SM, Jenkinson M, Woolrich MW, et al. (2004) Advances in functional and structural MR image analysis and implementation as FSL. Neuroimage 23: S208-S219.

Smith SM and Nichols TE (2009) Threshold-free cluster enhancement: Addressing problems of smoothing, threshold dependence and localisation in cluster inference. Neuroimage 44: 83-98.

Stone J, Kotoula V, Dietrich C, et al. (2015) Perceptual distortions and delusional thinking following ketamine administration are related to increased pharmacological MRI signal changes in the parietal lobe. $J$ Psychopharmacol 29: 1025-1028.
Terem I, Ni WW, Goubran M, et al. (2018) Revealing sub-voxel motions of brain tissue using phase-based amplified MRI (aMRI). Magnc Reson Med 80: 2549-2559.

Van Veen BD, Van Drongelen W, Yuchtman M, et al. (1997) Locali zation of brain electrical activity via linearly constrained minimum variance spatial filtering. IEEE Trans Biomed Eng 44: 867-880.

Winkler AM, Ridgway GR, Webster MA, et al. (2014) Permutation inference for the general linear model. Neuroimage 92: 381-397.

Woolrich MW, Jbabdi S, Patenaude B, et al. (2009) Bayesian analysis of neuroimaging data in FSL. Neuroimage 45: S173-S186.

Worsley K (2001) Statistical analysis of activation images. In: Jezzard P, Matthews PM and Smith SM (eds) Functional MRI: An Introduction to Methods. New York: Oxford University, pp. 251-270. 